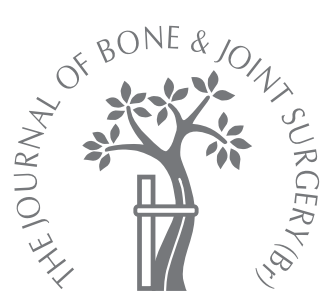

D. W. N. Simon, C. E. Clarkin, V. Das-Gupta, S. C. F. Rawlinson, R. J. Emery, A. A. Pitsillides

From St Mary's Hospital, London, England

\section{Identifying the cellular basis for re- implantation failure in repair of the rotator cuff}

D. W. N. Simon, MBBS, BSc, FRCS(Trauma \& Orth), Locum Consultant Orthopaedic

Surgeon

Department of Trauma and Orthopaedic Surgery King's College Hospital,

Denmark Hill, London SE5 9RS, UK.

ㅍ. C. E. Clarkin, BSc, PhD, Postdoctoral Research Assistant

IV V. Das-Gupta, BSc, PhD, Research Associate

I. C. F. Rawlinson, BSc, PhD, Assistant Lecturer

A. A. Pitsillides, $\mathrm{BSc}, \mathrm{PhD}$

Reader in Cell-Matrix Biology

Department of Veterinary Basic

Sciences

Royal Veterinary College, Royal

College Street, London NW1

OTU, UK.

n. J. Emery, MS, FRCS, Reader in Surgery Department of Trauma and Orthopaedic Surgery

St Mary's Hospital, Praed

Street, London W2 1NY, UK.

Correspondence should be sent to Mr D. W. N. Simon; e-mail: domsimon@doctors.org.uk

C)2008 British Editorial Society of Bone and Joint Surgery doi:10.1302/0301-620X.90B5. $20013 \$ 2.00$

$J$ Bone Joint Surg $[\mathrm{Br}]$ 2008;90-B:680-4.

Received 27 July 2007;

Accepted 8 January 2008

\begin{abstract}
We examined cultured osteoblasts derived from paired samples from the greater tuberosity and acromion from eight patients with large chronic tears of the rotator cuff.

We found that osteoblasts from the tuberosity had no apparent response to mechanical stimulation, whereas those derived from the acromion showed an increase in alkaline phosphatase activity and nitric oxide release which is normally a response of bone cells to mechanical strain. By contrast, we found that cells from both regions were able to respond to dexamethasone, a well-established promoter of osteoblastic differentiation, with the expected increase in alkaline phosphatase activity.

Our findings indicate that the failure of repair of the rotator cuff may be due, at least in part, to a compromised capacity for mechanoadaptation within the greater tuberosity. It remains to be seen whether this apparent decrease in the sensitivity of bone cells to mechanical stimulation is the specific consequence of the reduced load-bearing history of the greater tuberosity in these patients.
\end{abstract}

Tears of the rotator cuff are common. The incidence in human cadavers was found to be as high as $30 \% .^{1}$ Re-implantation of the ruptured tendon of supraspinatus into the greater tuberosity of the humerus fails in up to $50 \%$ of cases. ${ }^{2}$ A tear of the rotator cuff is often accompanied by thinning of the trabecular and cortical bone in the greater tuberosity as well as loss of the normal cortical contour. This would appear to be the result of disuse osteoporosis which, in turn, seems to be a factor contributing to the poor outcome of repairs of the rotator cuff. ${ }^{3}$ It is not known if the bone of the greater tuberosity in these patients has an impaired mechanoadaptive response to re-implantation of the tendon of supraspinatus, and, if so, whether this contributes to the high rate of failure.

It has been shown that healing of the bonetendon interface is mediated by cells originating in the subacromial bursa and the greater tuberosity, and not by cells from the tendon stump. ${ }^{4}$ It has become standard practice therefore to debride the stump to expose the tuberosity, but debate remains as to whether cortical or cancellous bone provides a better environment for reimplantation. Cole et $\mathrm{al}^{5}$ found that the properties of the tendon in chronic tears were inferior to those in acute tears and that larger tears had a reduced capacity for healing. Furthermore, the pro-inflammatory cells and mediators needed for healing were found to be more abundant in the tendon samples from small tears. Tendon samples from large tears show deposition of amyloid, oedema and degeneration, ${ }^{6}$ and cellular activity is lower in larger tears. ${ }^{7}$ These findings suggest that the healing of tendon to bone is likely to be impaired in larger chronic tears.

Failure of repair of the rotator cuff has also been attributed to the method of fixation. Most techniques use sutures or anchors and these have been studied extensively. ${ }^{8,9}$ Excessive tension in the repair may also result in failure. Reilly et $\mathrm{al}^{10}$ showed that if the arm was abducted after supraspinatus had been repaired, the force over the repair was reduced by $34 \mathrm{~N}$. The application of a force of $34 \mathrm{~N}$ across a cadaver repair for 24 hours caused considerable damage to that repair.

When repairing the rotator cuff, the tendon of supraspinatus is re-implanted into a trough in the greater tuberosity, and the bone which is removed to create this trough can be analysed. Acromioplasty is carried out at the same time as repair. Since the acromion is constantly loaded by the coracoacromial ligament and is resected during the operation, an opportunity presents itself to acquire a control sample from the same shoulder. ${ }^{11}$ The availability of these paired samples allows osteoblasts from each of these sites to be studied to determine whether any impairment of bone formation contributes to the failure of the repair. 

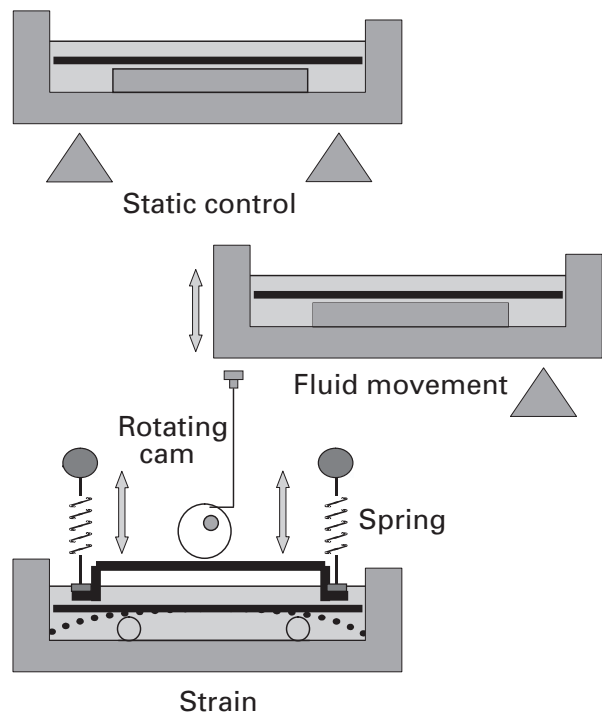

Fig. 1

Diagram of a custom-built apparatus used for applying strain to cells by subjecting plastic strips, on to which cells have been seeded, to four-point bending. As the eccentric cam rotates it presses the loading platen on to the vertical edges of the plastic strips and causes their deformation (to the dotted position) in an arc around the paired fulcra underlying the strips. The platen is returned to the unloaded position by the spring. The rotation of the cam also acts to rock the chamber into which strips subjected to fluid movement are placed. Static (undisturbed) controls remain in the incubator during the loading period.

The soluble autocoid, nitric oxide (NO), is released by osteoblasts in response to the application of dynamic mechanical strain. ${ }^{12,13}$ Its involvement in the adaptive response of bone to loading is supported by studies which have shown that non-selective inhibitors of NO-synthase suppress the osteogenic effect of loading in vivo. ${ }^{14,15}$ Strainrelated increases in osteoblast differentiation may also be assessed by measuring increases in cellular alkaline phosphatase activity. Strain-related NO release and increases in alkaline phosphatase activity from osteoblasts are therefore well-established markers of the bone-cell response to mechanical stimuli in vitro.

Dexamethasone is a synthetic glucocorticoid which promotes differentiation of osteoprogenitor cells into osteoblasts in vitro. Prolonged treatment of osteoblast cultures with dexamethasone increases cellular alkaline phosphatase activity and the formation of mineralised bone nodules in primary human osteoblasts. ${ }^{16}$ By subjecting primary human osteoblasts obtained from the 'normal' acromion and from the 'osteoporotic' greater tuberosity to controlled mechanical strain in vitro, we have examined whether an impaired response to mechanical stimulation contributes to the failure of the mechanoadaptive response of bone after repair of the rotator cuff.

\section{Patients and Methods}

Paired acromial and tuberosity bone samples were obtained intra-operatively from eight consecutive patients during open repair of the rotator cuff. There were four men and four women with a mean age of 58 years (55 to 80). Approval of the Ethical Committee and informed consent from each patient were obtained pre-operatively. The diagnosis of a chronic full-thickness tear was established by clinical examination, plain radiography and MRI. Patients with a tear of less than $3 \mathrm{~cm}$ in size were excluded. A sample of bone was taken from beneath the torn insertion of supraspinatus on the greater tuberosity. At operation the coracoacromial ligament was released and a Neer acromioplasty ${ }^{17}$ carried out using an oscillating saw. This provided a sample of bone from the anterior part of the acromion to act as a control.

Cell culture. Bone samples were collected under aseptic conditions and transported from the operating theatre in sterile phosphate-buffered saline (PBS). In the laboratory, all the soft tissues were carefully removed. The bone samples were then cut into fragments of approximately $1 \mathrm{~mm}^{2}$ and placed into $75 \mathrm{~mm}^{2}$ tissue culture flasks (Corning BV Life Sciences, Schipol, Netherlands) with Dulbecco's minimal essential medium (DMEM; Invitrogen, Paisley, United Kingdom) supplemented with $10 \%$ heat-inactivated fetal calf serum, $2 \mathrm{~mL}$ L-glutamine, $100 \mathrm{U} / \mathrm{ml}$ of penicillin and $100 \mathrm{mg} / \mathrm{ml}$ of streptomycin. These were maintained in a humidified $\mathrm{CO}_{2}$ incubator $(5 \%)$ at $37^{\circ} \mathrm{C}$ for 21 days to allow cell outgrowth. The media were replenished twice weekly. After 21 days, the bone fragments were removed and used to establish a second set of cultures. The cultures were washed with PBS and released by trypsin/ethylenediaminetetraacetic acid (EDTA). The cells were then re-suspended and seeded at a density of 2500 cells per $\mathrm{cm}^{2}$. The medium was changed twice weekly until the cells had reached confluence. $^{13}$

Application of mechanical strain to cultured primary osteoblasts. In order to prepare for mechanical strain testing, confluent osteoblast cultures from both the tuberosity and acromion were re-seeded onto sterile tissue-culture-treated plastic strips ( $25 \mathrm{~mm}$ by $75 \mathrm{~mm}$ ) at 125000 cells per $\mathrm{cm}^{2}$. The strips were subsequently maintained for 48 hours. Before applying mechanical strain according to the method of Pitsillides et al, ${ }^{12}$ fresh media containing $10 \%$ fetal calf serum was applied ( $9 \mathrm{ml}$ per strip, $27 \mathrm{ml}$ per $100 \mathrm{~mm}^{2}$ dish) and the cells on strips were returned to the incubator to equilibrate for 30 minutes. Six strips from each sample, with their adherent cells were transferred into the chambers of the loading jig (Fig. 1) along with $8 \mathrm{ml}$ of fresh equilibrated medium and then returned to the incubator $15 \%$ $\left.\mathrm{CO}_{2}, 37^{\circ} \mathrm{C}\right)$. Dynamic mechanical strains of $3400 \mu \varepsilon$ were applied with a frequency of $1 \mathrm{~Hz}$ for ten minutes, followed by a further ten-minute equilibration phase. Fluid movement alone was applied to six more strips in an identical jig for ten minutes to act as a control. Static (unperturbed) controls remained in the incubator for 20 minutes. 


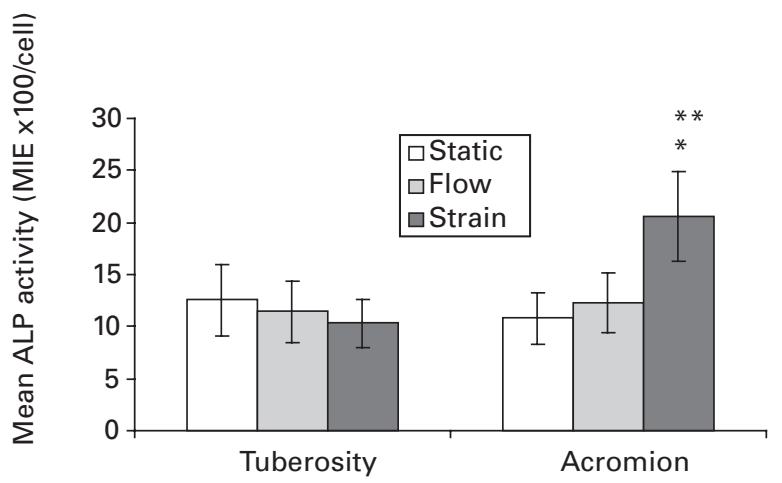

Fig. 2

Bar chart showing changes in alkaline phosphatase (ALP) activity (mean integrated extinction (MIE) $\times 100 /$ cell) induced 24 hours after the application of mechanical strain for ten minutes (flow control) to osteoblasts derived from the tuberosity and acromion $\left(^{*}\right.$, paired $t$-test $\mathrm{p}<0.003$ vs static; ${ }^{*}, \mathrm{p}<0.003$ vs flow).

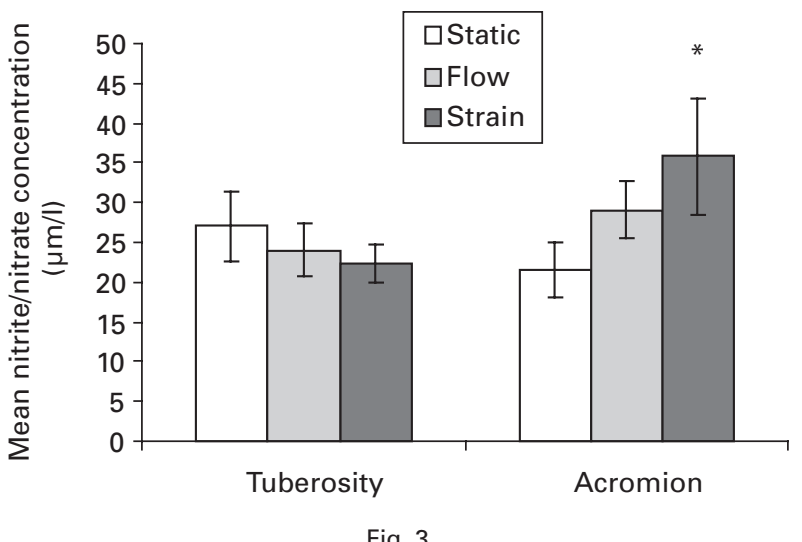

Bar chart showing change in total nitrite/nitrate concentration $(\mu \mathrm{M} / \mathrm{L})$ in the medium conditioned by osteoblast-like cells derived from the tuberosity and acromion ten minutes after application of mechanical strain for ten minutes (flow control) ${ }^{*}$, paired $t$-test $\mathrm{p}<0.05$ vs static control).
Measurement of nitric oxide concentration in the medium. Nitric oxide is rapidly converted in solution to the stable metabolites, nitrite and nitrate. Combined nitrite/nitrate levels were measured in duplicated medium samples stored at $-20^{\circ} \mathrm{C}$ before their analysis. Concentrations of nitrite/ nitrate in the samples were measured, in duplicate, using a commercially-available kit ( $\mathrm{R}$ and $\mathrm{D}$ Systems, Abingdon, United Kingdom).

Assessment of alkaline phosphatase activity per cell. To assess alkaline phosphatase activity, the osteoblasts were washed with PBS, fixed in ice-cold methanol and acetone $(50 / 50)$ for ten minutes, air dried and stored at $-80^{\circ} \mathrm{C}$ before reaction. The alkaline phosphatase activity was disclosed by incubation in reaction medium containing $1 \%$ Fast Blue RR salt (Sigma Ltd, Poole, United Kingdom) and 1\% napthol-AS-MX phosphate in $50 \mathrm{mM}$ Tris ( $\mathrm{pH}$ 9.2), for 30 minutes at $37^{\circ} \mathrm{C} .{ }^{18,19}$ After reaction, the cells were washed in distilled water, allowed to dry and mounted in Aquamount (Sigma).

The amount of reaction product, a measure of the alkaline phosphatase activity per cell, was measured in individual cells using a Vickers M85A scanning and integrating microdensitometer (Vickers Instruments, York, United Kingdom) which measured the optical density of the coloured reaction product in individual cells ${ }^{20,21}$ using a $\times$ 20 objective and an A6 mask with a scanning spot of $0.4 \mu \mathrm{m}$ diameter in the plane of the cell at $\lambda=585 \mathrm{~nm}$. Values were calculated as the mean integrated extinction $(\times 100)$ per cell in at least 50 cells in each well or plastic strip on to which cells had been seeded. At least three wells or strips were measured in each treatment group. Thus, the values of enzymic activity/cell were based on a mean of at least 150 cells. ${ }^{12}$ Assessment of the capacity for differentiation. We examined the response of cells derived from the acromion and tuberosity to treatment with the synthetic glucocorticoid, dexamethasone. The cells were cultured in a medium containing dexamethasone $(10 \mathrm{nM}), \mathrm{L}$-ascorbic acid $(50 \mu \mathrm{g} / \mathrm{ml})$ and $\beta$-glycerophosphate $(2 \mathrm{mg} / \mathrm{ml})$ for three days, then fixed and analysed for alkaline phosphatase activity, as described above.

Statistical analysis. Data were analysed by a paired (onetailed) $t$-test (SPSS Inc., Chicago, Illinois). A p-value $\leq 0.05$ was considered to be statistically significant.

\section{Results}

Previous studies have shown that both increases in mechanical loading in vivo and the application of direct mechanical strain to cells derived from normal bone in vitro induce the differentiation of osteoblasts. ${ }^{22,23}$ We found that acromial osteoblasts had statistically significant increases in alkaline phosphatase activity/cell when exposed to mechanical strain, but not in response to medium perturbation alone (Fig. 2). By contrast, osteoblasts derived from the tuberosity expressed similar alkaline phosphatase activity/cell to those derived from the acromion under static conditions, but did not show any significant increases in alkaline phosphatase activity/cell in response to the application of mechanical strain or medium perturbation (Fig. 2).

In keeping with previous findings, ${ }^{12,13}$ we found that acromial osteoblasts showed statistically significant increases in release of $\mathrm{NO}$ within ten minutes $(173 \%$ of control static levels) of the application of mechanical strain, but not in response to medium perturbation alone (flow, Fig. 3). The levels of osteoblastic NO release under static conditions was found not to differ significantly in cells from either the acromion or the humeral tuberosity (Fig. 3). Despite this, osteoblasts derived from the tuberosity were found not to show any significant increases in medium nitrite/nitrate accumulation in response to either flow or strain stimuli (Fig. 3). 


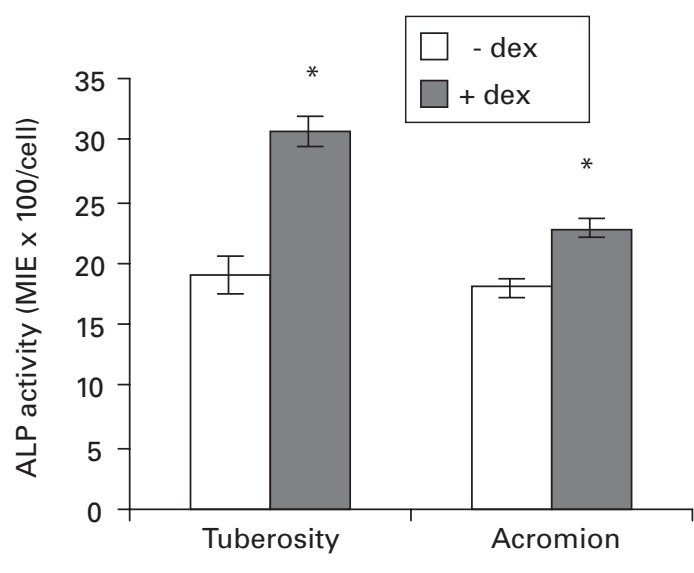

Fig. 4

Bar chart showing changes in alkaline phosphatase (ALP) activity (mean integrated extinction (MIE) $\times 100 /$ cell) induced 24 hours after treatment of osteoblasts derived from the tuberosity and acromion with dexamethasone (dex) (*, paired $t$-test $\mathrm{p}<0.01 \mathrm{vs}$ untreated control)

Our studies showed that unlike osteoblasts derived from the acromion, tuberosity-derived osteoblasts did not show increases in alkaline phosphatase activity/cell and had a modified NO release in response to mechanical stimuli. In order to determine whether this apparent resistance to strain-induced differentiation could be bypassed by an alternative inducer of differentiation, we examined the well-established stimulatory effect of dexamethasone on both acromial and tuberosity osteoblast-like cell behaviour. Using alkaline phosphatase activity to measure differentiation, our results showed that osteoblasts derived from both the tuberosity and the acromion (paired $t$-test $\mathrm{p}=0.01$ ) showed increases in alkaline phosphatase activity/cell in response to treatment with dexamethasone (Fig. 4). We found that tuberosity-derived osteoblasts had a significantly greater dexamethasone-induced increase in alkaline phosphatase activity/cell than acromion-derived osteoblasts (56\% difference), while untreated osteoblasts from either site had similar alkaline phosphatase activity (Fig. 4).

\section{Discussion}

It has been shown that cultured osteoblasts show increases in alkaline phosphatase activity in response to the application of mechanical strain both in vivo and in vitro. Our studies suggest that osteoblasts can adopt, and conserve in culture, a range of behaviour which reflects the loadbearing history of the site from which they have been derived. The behaviour of osteoblasts derived from sites of osteoporosis in which rates of bone formation are diminished, such as the greater tuberosity from patients with a tear of the rotator cuff, includes a selective loss in their capacity to release $\mathrm{NO}$ and to differentiate in response to the direct application of mechanical stimuli.
Rubin and Lanyon ${ }^{24}$ suggested that each bone has a genetically determined 'set point', below which it will not reduce its mass, however low its habitual strain exposure may fall. In the greater tuberosity, tension and compressive forces are large and relatively frequent. The skull has a high set point and hence disuse will affect it less than a long bone. It is therefore possible that the tuberosity has a low set point and that this underpins the osteoporosis which occurs at this site as a consequence of disuse. In greater tuberosities associated with tears of the rotator cuff, there is a gross reduction in trabecular bone and cortical thinning. ${ }^{3}$

In our study we have shown that bone cells from the tuberosity failed to show normal responses to mechanical strain. This appears to be balanced, however, by a significantly greater response to dexamethasone compared with that of cells derived from the acromion. This response may represent an upregulation of non-strain-related mechanisms to promote differentiation of the osteoblasts with reduced mechanosensitivity. If chemical signals promote differentiation but strain does not, it may be possible to promote osteogenesis by mimicking the down-stream consequences of mechanical strain. Establishing the mechanism by which cells respond to mechanical stimuli may allow the development of strategies to mimic the influence of strain at sites of disuse.

These data also support the existence of an apparent 'strain memory' in individual cells derived from two bone sites exposed to distinctly different loads in vivo. This carries with it the implication that a deficient response of bone cells to mechanical stimuli may also contribute to the failure of a repair of the rotator cuff. Bone cells from within the greater tuberosity may fail to respond to the changing mechanical demands of tendon re-implantation. Better outcomes may be achieved if repair of the tendon is attempted early before bone loss occurs from disuse. This does, however, raise the question of how fast bone loss occurs both quantitatively and qualitatively and whether there is scope for slowing or reversing this process.

No benefits in any form have been received or will be received from a commercial party related directly or indirectly to the subject of this article.

\section{References}

1. Reilly P, Macleod I, Macfarlane R, Windley J, Emery RJ. Dead men and radiologists don't lie: a review of cadaveric and radiological studies of rotator cuff tear prevalence. Ann $R$ Coll Surg Eng/ 2006;88:116-21.

2. Harryman D, Mack L, Wang K, et al. Repairs of the rotator cuff: correlation of functional results with integrity of the cuff. J Bone Joint Surg [Am]1991;73-A:982-9.

3. Meyer D, Fucentese S, Koller B, Gerber C. Association of osteopenia of the humeral head with full-thickness rotator cuff tears. J Shoulder Elbow Surg 2004;13:333-7.

4. Uhthoff H, Sano H, Trudel G, Ishii H. Early reactions after reimplantation of the tendon of supraspinatus into bone: a study in rabbits. J Bone Joint Surg [Br] 2000;82-B:1072-6.

5. Cole B, Rodeo S, O'Brien S, et al. The anatomy and histology of the rotator interval capsule of the shoulder. Clin Orthop 2001;390:129-37.

6. Matthews T, Hand G, Rees J, Athanasou N, Carr A. Pathology of the torn rotator cuff tendon: reduction in potential for repair as tear size increases. J Bone Joint Surg [Br] 2006;88-B:489-95.

7. Matthews TJW, Smith SR, Peach CA, et al. In vivomeasurement of tissue metabolism in tendons of the rotator cuff: implications for surgical management. J Bone Joint Surg [Br] 2007;89-B:633-8. 
8. Schneeberger AG, von Roll A, Kalberer F, Jacob HA, Gerber C. Mechanical strength of arthroscopic rotator cuff repair techniques: an in vitro study. J Bone Joint Surg [Am] 2002;84-A:2152-60.

9. Burkhart SS, Lo IK. Arthroscopic rotator cuff repair. J Am Acad Orthop Surg 2006;14:333 46.

10. Reilly P, Bull A, Amis A, et al. Passive tension and gap formation of rotator cuff repairs. J Shoulder Elbow Surg 2004;13:664-7.

11. Chambler A, Bull A, Reilly P, Amis A, Emery R. Coracoacromial ligament tension in vivo. J Shoulder Elbow Surg 2003;12:365-7.

12. Pitsillides A, Rawlinson S, Suswillo R, et al. Mechanical strain-induced NO production by bone cells: a possible role in adaptive bone (re)modeling? FASEB J1995;9:1614-22.

13. Zaman G, Pitsillides A, Rawlinson S, et al. Mechanical strain stimulates nitric oxide production by rapid activation of endothelial nitric oxide synthase in osteocytes. J Bone Miner Res 1999;14:1123-31.

14. Fox $\mathbf{S}$, Chambers $\mathrm{T}$, Chow J. Nitric oxide is an early mediator of the increase in bone formation by mechanical stimulation. Am J Physio/ 1996;270:955-60.

15. Turner C, Takano Y, Owan I, Murrell G. Nitrix oxide inhibitor L-NAME suppresses mechanically induced bone formation in rats. Am J Physiol 1996;270:634-9.

16. Yao KL, Todescan RJ, Sodek J. Temporal changes in matrix protein synthesis and mRNA expression during mineralized tissue formation by adult rate bone marrow cells in culture. J Bone Miner Res 1994:9:231-40.
17. Neer C. Anterior acromioplasty for the chronic impingement syndrome in the shoulder: a preliminary report. J Bone Joint Surg [Am] 1972;54-A:41-50.

18. Dodds RA, Ali N, Pead MJ, Lanyon LE. Early loading-related changes in the activity of glucose 6-phosphate dehydrogenase and alkaline phosphatase in osteocytes and periosteal osteoblasts in rat fibulae in vivo. J Bone Miner Res 1993;8:261-7.

19. Bradbeer JN, Lindsay PC, Reeve J. Fluctuation of mineral apposition rate at individual bone-remodeling sites in human iliac cancellous bone: independent correlations with osteoid width and osteoblastic alkaline phosphatase activity. J Bone Miner Res 1994;9:1679-86.

20. Chambler A, Rawlinson S, Emery R, Pitsillides A. Quantitative cytochemical evidence for local increases in bone turnover at the acromial enthesis of the human coracoacromial ligament. J Rheumato/ 2004;31:2216-25.

21. Chayen J. Quantitative cytochemistry: a precise form of cellular biochemistry. Biochem Soc Trans 1984;12:887-98.

22. Rubin C, Lanyon L. Regulation of bone formation by applied dynamic loads. J Bone Joint Surg [Am] 1984;66-A:397-402.

23. Lanyon L. Functional strain in bone tissue as an objective, and controlling stimulus for adaptive bone remodelling. J Biomech 1987;20:1083-93.

24. Rubin C, Lanyon L. Osteoregulatory nature of mechanical stimuli: function as a determinant for adaptive remodeling in bone. J Orthop Res 1987:5:200-10. 